\title{
Development of An Efficient Algorithm to Enumerate the Number of Constitutional Isomers of Alkyne Series
}

\author{
Mahesh Kumar Chouhan \\ Dept. of Computer Science and Engineering \\ Dr. B. R. Ambedkar National Institute of \\ Technology \\ Jalandhar, India
}

\author{
A. L . Sangal \\ Dept. of Computer Science and Engineering \\ Dr. B. R. Ambedkar National Institute of \\ Technology \\ Jalandhar, India
}

\begin{abstract}
Computerized Enumeration of alkane's isomers has long been a topic of interest to the researchers. The double bond carbon content and triple bond carbon content isomers were mostly neglected for many decades by the researchers and claimed in the literature that there is no simple algorithm for these problems. In this paper an efficient and simple algorithm has been proposed to count the number of constitutional isomers of alkynes series. By using this recursive algorithm the resulting computation of number of isomers for alkynes with any number of carbon contents [limited to system specifications] are easily enumerated. Further the time complexity of the proposed algorithm has also been investigated. The algorithm has been implemented using object oriented programming language-Java.
\end{abstract}

\section{Keywords}

Carbon, Isomer, Counting,alkynes,enumeration,time complexity

\section{INTRODUCTION}

Before outlining the background and scope of the problem that we have consider, it will be appropriate to review briefly the basic facts about chemical compounds. For our purpose we can regard a molecule of a chemical compound as an assemblage of atoms in which some atoms are linked to other by "valency bonds". These may be single, double and triple. In this paper we shall be concerned only about hydrocarbons. A hydrocarbon is any chemical compound containing only carbon and hydrogen. The carbon atom has valency four and the hydrogen one. Hydrocarbons can be classified into acyclic and cyclic types. In this paper we have discussed only acyclic type. The acyclic hydrocarbons are characterized by a branched tree structure, and can be separated into three most categories [3].

Alkanes: Contain single bonds, and have the general formula $\mathrm{C}_{\mathrm{n}} \mathrm{H}_{2 \mathrm{n}+2 \text {. }}$.

$>$ Alkenes Contain double bonds, and have the general formula $\mathrm{C}_{\mathrm{n}} \mathrm{H}_{2 \mathrm{n}}$.

$>$ Alkynes contain a triple bond, and have the general formula $\mathrm{C}_{\mathrm{n}} \mathrm{H}_{2 \mathrm{n}-2}$.

Isomers are any set of chemical compounds that have the same chemical formula, but a different arrangement of the atoms. For example pentanes have the three isomers.

\section{RELATED WORK}

In the past a Simple algorithm for counting constitutional isomers of alkanes, single $\mathrm{C}$-atom isotopically labelled alkanes, and monosubstituted alkanes was reported[4] and the algorithm was implemented in a high level programing language-Turbo Pascal. Mathematically recount of alkenes with geometric isomerism due to a double bond was taken into consideration[5] Further the mathematical relationship between the number of isomers of alkenes and alkynes was established from the enumeration of isomers of alkenes and alkynes[2].Computerised isomer Enumeration of the Alkane Series was presented[1] and an algorithm to enumerate the isomers of eighteen carbon atoms alkane series was implemented in the Object Oriented programing language- $\mathrm{C}++$.

\section{THE ALGORITHM}

\subsection{Process}

The main goal of this algorithm is to enumerate the isomers of any number of carbons of alkynes series in an accurate and efficient manner The proosed algorithm is using recursion and has been doveloped using an object oriented programing language-Java.A custom data structure has been used in this program.

\subsection{Method}

The algorithm is based on the formation of alkyl biradicals [8] These alkyl biradicals can be imagined to be formed by a hypothetical chemical reaction in which an alkane molecule loses two protons and becomes an alkyl biradical, i.e. is with two unpaired electrons. Interestingly, not all possible alkane isomers are required to form all possible alkyl biradicals as alkane molecule has many different sites where the two protons can be lost. When a single alkyl biradical combines with another alkyl biradical, the four electrons will be paired up and double bond will be formed [2].

\subsection{Procedure}

ALKYNES_ISOMER ( $n$, Count $=0$, Ptr, nofcarbon) $n=$ number

of isomeric alcohols, nofcarbon=no of carbon of alkynes, count is number of isomer of alkenes

1.Initialize array $\{n 1, n 2, n 3$... $. n i\}$

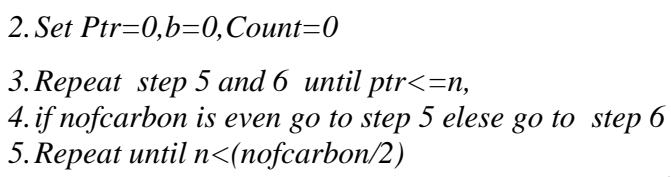

I. Count=Count+araay $[n] *$ array $[$ nofcar bon- $(n+1)]$ 
II. if ( $n==$ nofcarban/2) go to step (a) else go to step (lll).
a) Break
b) Return Count
c) Exit

III. $\quad$ Ptr $=P t r+1$

6. Repeat step (I) to () until $n<$ (nofcarbon- $n$ )

I. if $(n==($ nofcarbon-1)/2) go to step (II) else go to step (lll)

II. Count $=b+(\operatorname{array}[n] *(\operatorname{array}[n]+1)) / 2$

a) break

b) Return Count and exit

III. $\quad b=b+\operatorname{array}[n] * \operatorname{array}[$ nofcarbon- $(n-1)]$

IV. $\quad$ Ptr $=$ Ptr +1

V. Return Count

VI. Exit

\section{RESULT AND DISCUSSION}

\subsection{Outputs}

The enumeration of isomer is not only of particular interest in theoretical chemistry, but also expanding field of computational chemistry.

The procedure described in section III $(\mathrm{C})$ will enumerate the isomers of any member of the alkynes family exhaustively and irredundantly. In this research works using the said procedure isomers of alkynes series with carbon content from 1 to 47 have been calculated without any error. Isomers of higher carbon content could not be calculated because of system limitation.

Table 1 Alkynes Isomes

\begin{tabular}{|c|c|}
\hline $\begin{array}{c}\text { Number } \\
\text { of carbon }\end{array}$ & $\begin{array}{c}\text { Number of constitutional Isomer } \\
\text { of alkynes series }\end{array}$ \\
\hline 1 & 1 \\
\hline 2 & 1 \\
\hline 3 & 2 \\
\hline 4 & 3 \\
\hline 5 & 7 \\
\hline 6 & 14 \\
\hline 7 & 32 \\
\hline 8 & 72 \\
\hline 9 & 171 \\
\hline 10 & 405 \\
\hline 11 & 989 \\
\hline 12 & 2426 \\
\hline 13 & 6045 \\
\hline 14 & 15167 \\
\hline 15 & 38422 \\
\hline
\end{tabular}

\begin{tabular}{|c|c|}
\hline 16 & 97925 \\
\hline 17 & 251275 \\
\hline 18 & 648061 \\
\hline 19 & 1679869 \\
\hline 20 & 4372872 \\
\hline 21 & 11428365 \\
\hline 22 & 29972078 \\
\hline 23 & 78859809 \\
\hline 24 & 208094977 \\
\hline 25 & 550603722 \\
\hline 26 & 1460457242 \\
\hline 27 & 3882682803 \\
\hline 28 & 10344102122 \\
\hline 29 & 27612603765 \\
\hline 30 & 73844151259 \\
\hline 31 & 197818389539 \\
\hline 32 & 530775701520 \\
\hline 33 & 1426284383289 \\
\hline 34 & 3838066701350 \\
\hline 35 & 10341758769406 \\
\hline 36 & 27900947721908 \\
\hline 37 & 75362644825968 \\
\hline 38 & 203787850635992 \\
\hline 39 & 551645375673949 \\
\hline 40 & 1494781478155753 \\
\hline 41 & 4054242571711886 \\
\hline 42 & 11006161817116528 \\
\hline 43 & 29904564722290758 \\
\hline 44 & 81319947893937569 \\
\hline 45 & 221308699013145314 \\
\hline 45 & 602735147429051222 \\
\hline 47 & 1642733167881428721 \\
\hline
\end{tabular}




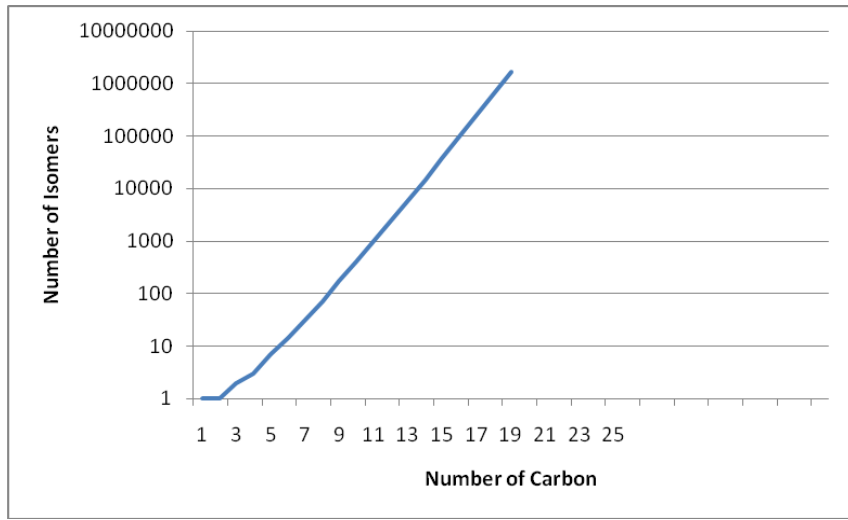

Figure 1

Figure 1 show that, after the carbon content eight, the graph takes on a linear outward show, which indicates that the number of isomers increases logarithmically with the carbon content. Before eight however, there does not appear a clear cut pattern or relationship between numbers of carbon contents with its isomers however beyond carbon content eight there is a linear relationship which could be used to estimate the number of isomers for higher carbon contents.

\subsection{PERFORMANCE OF THALGORITHEM}

\subsubsection{Accuracy}

The developed algorithm has produced the number of isomer exhaustively irredundantly up to carbon contents 47 without any error as compare to the published results results[2] hence the proposed algorithm is accurate .

\subsubsection{Time Complexity \& Efficiency:}

The algorithm denotes a significant improvement over previous algorithms developed earlier in respect of computation time. The time complexity of this algorithm is by far its strongest point. As established in the figure 2, the amount of computational time required varies approximation linearly with the number of carbons. This is notable because in past algorithms, computational time have increased logarithmically or greater, with the increasing number of isomers. The time complexity is dependent on the creativity of a programmer and system configuration.

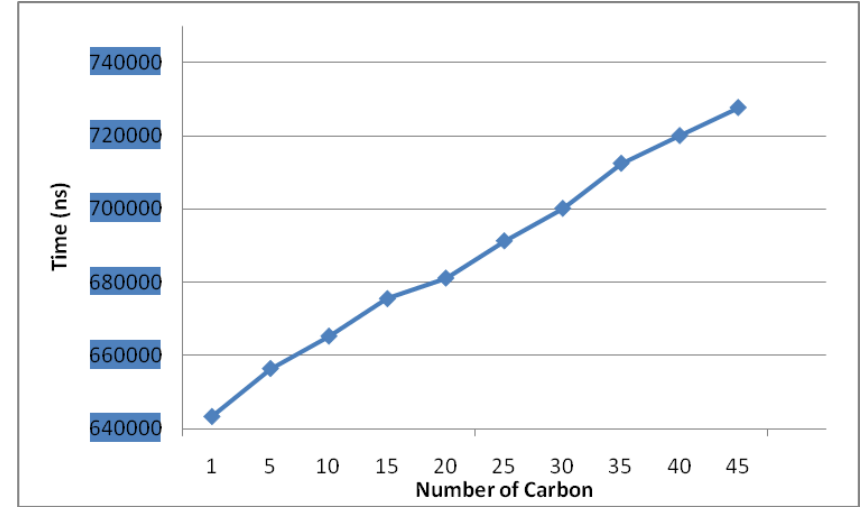

Figure 2

\section{CONCLUSIONS AND FUTURE SCOPE}

The developed algorithm has been successful in accomplishing the main goals of the current research work. However isomers of higher carbon contents could not be calculated because of system limitations.

The algorithm can be modified to enumerate the isomers of alkenes series with great accuracy

\section{REFERENCES}

[1] Kevin Ballard, Advisor M. Eicher and Mentor N. Preyer, “ Computerized Isomer Enumeration of the Alkane Series" 2003

[2] Ching-Wan Lam, "A mathematical relationship between the number of isomers of alkenes: Result established from the enumeration of isomer of alkenes from alkyl biradicals" J. of Mathematical Chemistry, 23, 1998,pp. 421-428.

[3] Ronald C. Read," Some recent results in chemical enumeration," Vol. 303,1972,pp.243-259.

[4] Chin-yah Yeh,"'Isomer Enummeration of Alkanes, Labeled Alkanes and Monosubstituted Alkanes" J. Chem. Inf. Computer Science, 35.1995,912-13.

[5] Chin-yah Yeh, Isomer Enumeration of Alkenes and Aliphatic Cyclopropane Derivatives. American Chemical Society,1996.

[6] C. C. Davish, K. Cross and M. Ebel, "Computer calculation of alkane isomer, “ J. Che. Educ. 48,1971,pp. 605.

[7] H. R. Henze and C. M. Blair, "The number of structurally isomeric alcohols of the methanol series, " J. Am. Chem. Soc. 53,1931,pp.3042-3046.

[8] R.C. Read, "The enumeration of acyclic chemica compound, in : Chemical Application of Graph Theory, ed. A. T. Balaban ,Academic Press, New York, 1976, pp. 25-61 